\title{
Optimization of the "in-silico" mate-pair method improved contiguity and accuracy of genome assembly
}

\author{
Tao Zhou ${ }^{1}$, Liang $\mathrm{Lu}^{1}$, and Chenhong $\mathrm{Li}^{1}$ \\ ${ }^{1}$ Shanghai Ocean University
}

September 25, 2021

\begin{abstract}
A combination of next-generation sequencing technologies and mate-pair libraries of large insert sizes is used as a standard method to generate genome assemblies with high contiguity. The third-generation sequencing techniques also are used to improve the quality of assembled genomes. However, both mate-pair libraries and the third-generation libraries require highmolecular-weight DNA, making the use of these libraries inappropriate for samples with only degraded DNA. An in silico method that generates mate-pair libraries using a reference genome was devised for the task of assembling target genomes. Although the contiguity and completeness of assembled genomes were significantly improved by this method, a high level of errors manifested in the assembly, further to which the methods for using reference genomes were not optimized. Here, we tested different strategies for using reference genomes to generate in silico mate-pairs. The results showed that using a closely related reference genome from the same genus was more effective than using divergent references. Conservation of in silico mate-pairs by comparing two references and using those to guide genome assembly reduced the number of misassemblies $(18.6 \%$ $-46.1 \%)$ and increased the contiguity of assembled genomes $(9.7 \%-70.7 \%)$, while maintaining gene completeness at a level that was either similar or marginally lower than that obtained via the current method. Finally, we compared the optimized method with another reference-guided assembler, RaGOO. We found that RaGOO produced longer scaffolds (17.8 Mbp vs 3.0 $\mathrm{Mbp}$ ), but resulted in a much higher misassembly rate (85.68\%) than our optimized in silico mate-pair method.
\end{abstract}

\section{Optimization of the "in-silico" mate-pair method improved contiguity and accuracy of genome} assembly

Tao Zhou ${ }^{1,2}$, Liang $\mathrm{Lu}^{1,2}$, Chenhong $\mathrm{Li}^{1,2, *}$

${ }^{1}$ Shanghai Universities Key Laboratory of Marine Animal Taxonomy and Evolution, Shanghai Ocean University, Shanghai, China 201306;

2 Shanghai Collaborative Innovation for Aquatic Animal Genetics and Breeding, Shanghai Ocean University, Shanghai 201306, China

*Corresponding author, chli@shou.edu.cn

\section{Abstract}

A combination of next-generation sequencing technologies and mate-pair libraries of large insert sizes is used as a standard method to generate genome assemblies with high contiguity. The third-generation sequencing techniques also are used to improve the quality of assembled genomes. However, both mate-pair libraries and the third-generation libraries require high-molecular-weight DNA, making the use of these libraries inappropriate for samples with only degraded DNA. An in silicomethod that generates mate-pair libraries using a reference genome was devised for the task of assembling target genomes. Although the contiguity and completeness of assembled genomes were significantly improved by this method, a high level of errors manifested in the assembly, further to which the methods for using reference genomes were not optimized. 
Here, we tested different strategies for using reference genomes to generate in silico mate-pairs. The results showed that using a closely related reference genome from the same genus was more effective than using divergent references. Conservation of in silicomate-pairs by comparing two references and using those to guide genome assembly reduced the number of misassemblies $(18.6 \%-46.1 \%)$ and increased the contiguity of assembled genomes $(9.7 \%-70.7 \%)$, while maintaining gene completeness at a level that was either similar or marginally lower than that obtained via the current method. Finally, we compared the optimized method with another reference-guided assembler, RaGOO. We found that RaGOO produced longer scaffolds (17.8 Mbp vs $3.0 \mathrm{Mbp})$, but resulted in a much higher misassembly rate (85.68\%) than our optimized in silico mate-pair method.

\section{KEY WORDS}

Genome assembly, in silico mate-pair, contiguity, accuracy, degraded DNA

\section{1 | INTRODUCTION}

Advances made in DNA sequencing during the past decade, has led to genomes of diverse organisms being successfully sequenced and assembled (de Man et al., 2016; Iorizzo et al., 2016; Jarvis et al., 2017; Lien et al., 2016). High-quality genome assembly requires high levels of contiguity, which enable new insights into genome structure evolution and increase the gene space completeness of the assembly (Berlin et al., 2015; Gordon et al., 2016; Koren et al., 2013; Loman, Quick, \& Simpson, 2015). However, the presence of repetitive regions in a genome poses a major challenge to the assembling of highly contiguous genomes. Mate-pair sequencing involves the generation of long-insert paired-end DNA libraries that span several kilobase pairs of long repeat regions. This is useful for many sequencing applications, including de novo sequencing, genome finishing, structural variant detection, and identification of complex genomic rearrangements (Maretty et al., 2017; Smadbeck et al., 2018; Tan, Tan, \& Cheng, 2020; van Heesch et al., 2013; Wetzel, Kingsford, \& Pop, 2011). During mate-pair library preparation, DNA is fragmented allowing DNA of a desired length to be isolated. Afterwards, the ends of the DNA fragments are biotinylated and circularized. Then, the DNA ring is sheared into smaller fragments (400-600 bp). Biotinylated fragments are enriched (by biotin tag), and adapters ligated. These are then ready for cluster generation and sequencing. Although this technology does not produce long reads, it is able to span repeat regions if the insert size is sufficiently large. Combining data generated from mate-pair library sequencing with those from short-insert paired-end reads provides a powerful combination of read lengths for maximal sequencing coverage across the genome, leading to a dramatic improvement in the assembly of large genomes. Mate pairs with small, medium, and large insert sizes are usually used to scaffold contigs in order to improve genome assemblies (Pop, Phillippy, Delcher, \& Salzberg, 2004).

Third-generation long-read sequencing technologies, such as PacBio (Rhoads \& Au, 2015) and Nanopore, (Jain, Olsen, Paten, \& Akeson, 2016), increase read lengths to overcome the challenge of sequencing repetitive regions that reads must be long enough to anchor in nonrepetitive sequences and span across the repeats. Repeats may be spanned, and subsequent assembling of the region is possible if the read length is substantially longer than the repeat region (Bongartz, 2019). Third-generation long reads are also used for scaffolding during genome assembly (Boetzer \& Pirovano, 2014).

High-quality DNA, which is crucial for mate-pair sequencing, can only be obtained from material that is both fresh and abundant. Similarly, high-molecular-weight DNA (>50 kb) is needed to realize the full beneficial effects of potential third-generation sequencing. The lack of suitable starting material limits the choice of sequencing technology and affects the quality of the obtained data. For example, in a comparative genomics study of ruminants, only the genomes of several species, such as mountain nyala, common eland, bongo, and oribi could be assembled at the contig level due to degenerate DNA samples, which were not suitable for constructing mate pair libraries (Chen et al., 2019). Another example of poor DNA involves studies of ancient DNA (aDNA) (Stoneking \& Krause, 2011) which mostly contains very short fragments between 44 and 172 bp (Sawyer, Krause, Guschanski, Savolainen, \& Paabo, 2012).

Although it is impossible to apply mate-pair or third generation sequencing to degenerate or ancient samples, 
(Grau, Hackl, Koepfli, \& Hofreiter, 2018) invented a method that generates in silicomate-pair libraries using a reference genome from a closely related species, thereby helping to assemble genomes at the scaffold level. In order to improve genome contiguity, they developed cross-species scaffolding - a new pipeline that imports long-range distance information directly into a de novo assembly process by constructing mate-pair libraries in silico . After processing, cleaned reads of target species were mapped to the repeat-masked reference genome, and consensus is computed. Next, read pairs of mate-pair libraries are generated based on consensus. Finally, the cleaned reads and in silico mate pairs are used to assemble the genome using SOAPdenovo2 (Luo et al., 2012). Application of thisin silico mate-pair method resulted in a dramatic improvement in contiguity and accuracy, as demonstrated by the assembling of two primate genomes, based on just 30x coverage of shotgun sequencing data (Grau et al., 2018). A drawback of this approach is the introduction of assembly chimeras (Grau et al., 2018). Furthermore, phylogenetic distance, quality, and completeness of the reference genome, as well as its overall synteny and transposable element content, influence the final number of misassemblies. Methods via which misassemblies can be reduced and best references can be chosen to generate in silicomate pairs are yet to be tested.

In addition to the in silico mate-pair method, referred to as the reference-guided approach, similarity between the target and reference species can also be made use of to gain additional information, which often leads to more complete and improved genome assemblies (Bao, Jiang, \& Girke, 2014; Pop et al., 2004; Schneeberger et al., 2011). In contrast to the in silico method that generates mate pairs prior to genome assembly, reference guide approaches, such as Chromosomer (Tamazian et al., 2016), Ragout (Kolmogorov, Raney, Paten, \& Pham, 2014), and RaGOO (Alonge et al., 2019) , use a single reference to order, orientate, and join contigs and long reads. Therefore, thein silico mate-pair method is more flexible than the reference guide approach. For example, high-quality, conserved mate pairs can be selected by comparing two or more reference genomes to reduce misassemblies in the target genome assembly.

In this study, we attempted to optimize the use of the in silicomethod. First, we investigated how the phylogenetic distance between a reference and a target affects the quality of genome assembly. We then tested whether generating a conserved mate pair by comparing multiple reference genomes improves the quality of genome assembly. Finally, we tested the effect of the optimized in silico mate-pair strategy on degraded samples and a simulated ancient DNA data.

\section{2 | MATERIALS AND METHODS}

\section{1 | Experimental design}

We designed three experiments using the published data and simulations. First, we tested the effect of using references with different phylogenetic distances to target species, on the quality of target genome assemblies, using the paired-end data of the walking catfish (Clarias batrachus) and a puffer fish (Takifugu bimaculatus ) (Table S1). For C. batrachus, genomes of two species, C. magur and C. macrocephalus, from the same genus, and one species, Ameiurus melas, from a different family but the same order, were selected as references. For T. bimaculatus, reference genomes of two species, T. rubripes and T. flavidus from the same genus, one species, Tetradon nigroviridis, from a different genus but the same family, and one species, Mola mola, from a different family but the same order, were selected. Secondly, we optimized the in silico mate-pair method by searching for conserved mate pairs generated using two or more references (Fig. 1) and used them to assemble the genomes via SOAPdenovo2 (Luo et al., 2012). Thirdly, we tested whether the optimized in silico method significantly improved the genome assembly of the mountain nyala (Tragelaphus buxtoni ), a highly degraded sample. Genomes of two species, T. scriptus and T. strepsiceros, from the same genus, one species, Bos grunniens, from a different genus but the same family, and one species,Moschus moschiferus, from a different family but the same order, were selected as references to produce in silico mate pairs for the purpose of assembling the genome of T. buxtoni . Lastly, we simulated single-end ancient DNA reads using $T$. flavidussequencing data to test the optimized in silico method and compared it with a reference-guided approach, RaGOO.

\section{2 | Data for the target species and references}


Raw data (fastq files) of the target species, C. batrachus ,T. bimaculatus, T. flavidus, and T. buxtoni were downloaded from the ENA database website (https://www.ebi.ac.uk/ena/browser/home, SRR7440020, SRR8285222, SRR7881551, SRR6913452, SRR6913453, SRR6913455). PCR duplicates were deleted using Prinseq (Schmieder \& Edwards, 2011). Adapters and low-quality bases were removed using Trim Galore (https://github.com/FelixKrueger/TrimGalore). Next, the reads were corrected using k-mers with BFC (Li, 2015). Multiplicity distribution of the 23-mers was counted using Jellyfish2 (Marçais \& Kingsford, 2011) and genome coverage was estimated using KrATER (https://github.com/mahajrod/KrATER). After processing, the final genome coverage of C. batrachus, T. bimaculatus, T. buxtoni, and simulated ancient DNA clean reads were all more than $30 \mathrm{x}$ (Table S2). The insert sizes of paired-end reads were $180 \mathrm{bp}, 300 \mathrm{bp}, 250 \mathrm{bp}$, $350 \mathrm{bp}$, for C. batrachus, T. bimaculatus, T. flavidus, and T. buxtoni, respectively.

Reference genome assemblies of C. macrocephalus, A. melas, T. rubripes, T. flavidus, T. nigroviridis, T. bimaculatus, M. mola, T. scriptus, T. strepsiceros , B. grunniens, and M. moschiferus were downloaded from the National Center for Biotechnology Information (NCBI); (Table S3-S5). The repeat contents of these genomes were masked using RepeatMasker (http://repeatmasker.org/).

\section{3 | Generating in silico mate-pair libraries using the original pipeline}

Multiple sets of in silico mate pairs were generated using the original in silico pipeline "cross-mates" (Fig. 2); (Grau et al., 2018). First, reads of the target organism were mapped onto the repeat-masked reference genome using BWA-MEM (Li, 2013) and default settings. A consensus was then computed using samtools/bcftools with the samtools legacy variant calling model ( $\mathrm{Li}, 2011$ ). Read pairs (mate pairs) were sampled from the consensus in systematic mode, that is, using exact insert sizes and sampling fragments at regularly spaced offsets, and skipping regions of coverage lower than three. For the test assemblies, in silico mate pairs were generated with at least 30x coverage each, with multiple insert sizes ranging from $500 \mathrm{bp}$ to $200 \mathrm{~Kb}$ (500 bp, $1 \mathrm{~Kb}, 1.5 \mathrm{~kb}, 2 \mathrm{~Kb}, 5 \mathrm{~Kb}, 10 \mathrm{~Kb}, 20 \mathrm{~Kb}, 50 \mathrm{~Kb}, 100 \mathrm{~Kb}, 200 \mathrm{~Kb}$ ). The in silico mate pairs generated using reference genomes from different grades of taxonomy were named as 'species name*'.

\section{4 | Optimizing the method by searching conservedin silico mate pairs}

We used a map method to search for conserved in silico mate pairs (Fig. 3). First, mate-pair reads generated using the first reference were mapped to another reference with BWA-MEM (Li, 2013) and default settings, as described above. Then, an in-house python script (Sam2fq.py) was used to select the mate-pair reads mapped within 20 percent deviation of insert sizes and in the same direction (not reversed). To distinguish conserved mate pairs generated from the original in silico method, these were named as 'species1-species2**' using two reference genomes, 'species1-species2-species $3^{* *}$ ' using three reference genomes, and 'species1-species2species3-species $4^{* *}$, using four reference genomes.

\section{5 | Simulation of ancient DNA reads}

To investigate the efficacy of the optimized in silico method in regard to genome assembly of extinct species with ancient DNA, we simulated ancient DNA reads. We chose the cleaned data of T. flavidus to simulate ancient DNA data because it is a high-quality genome assembly generated using both mate-pair sequencing and PacBio sequencing. After correction, the forward strand of paired-end reads (insert size of $250 \mathrm{bp}$, read length $150 \mathrm{bp}$ ) was cut at a random length to form $80 \mathrm{bp}$ to $100 \mathrm{bp}$ single-end reads using an in-house python script (Simulate.py). The size distribution of the simulated reads is shown (Fig. S1). For simulated ancient DNA, genomes of T. rubripes(same genus), T. bimaculatus (same genus), T. nivigroviridis (same family), and M. mola (same order) were selected as references. The statistics of these references are summarized (Table S6).

\section{6 | Genome assembly}

Following the pipeline of (Grau et al., 2018), de novo assembly of the target species genomes with in silico paired-ends and mate-pair reads were performed using SOAPdenovo2 (Luo et al., 2012). Firstly, the sparse pre-graph module was applied to use paired-end or simulated ancient DNA reads during de Brujin graph construction with the parameters, -g 15 -d 4 -e 4 -R -r 0, and parameter -M 1, during the contig phase. 
Secondly, in silico mate-pair reads generated by the original or optimized in silico method were mapped to contigs. Third, unique contigs were joined to scaffolds using mapped paired-end and mate-pair read information. For comparison with our optimized in silico mate-pair methods, we also used the RaGOO pipeline to perform genome assembly using the simulated ancient DNA reads with the following parameters: -f 1000 -d 100000 -i 0.2 -a 0.5 -s 0.5 -r -g 100 -m 10000. Unlike scaffolding by SOAPdenovo, the contigs produced by SOAPdenovo were ordered and oriented using RaGOO.

\section{7 | Evaluation of genome assembly}

Contiguity, misassemblies, and other assembly statistics were evaluated using Quast, which provides the maximum amount of information regarding assemblies (Gurevich, Saveliev, Vyahhi, \& Tesler, 2013). Completeness of the assemblies was measured by searching for 3,354 vertebrate orthologs in a set of protein predictions generated by Augustus, as implemented in BUSCO (Simao, Waterhouse, Ioannidis, Kriventseva, \& Zdobnov, 2015). Consistent regions between the resulting genome assembly and the "real" genome sequence, the best assembly based on experimental mate pairs or third-generation long reads, were identified using Mummer4 (Marcais et al., 2018) and then synteny between these were visualized using R (https://www.r-project.org/).

\section{3 | RESULTS}

\subsection{Number of in silico mate-pair libraries using single or multiple references}

Mate-pair libraries were generated using multiple reference genomes from the same genus, family, and order of target species. The quantities of mate-pair read pairs were counted (Table S7-S8). Referring to mate pairs generated for $C$. batrachus, the maximum number of in silico mate-pair reads was generated using C. magur (600,254,032, same genus) as a reference, and even more using the C. macrocephalus genome as a reference (349,115,222, same genus). Using A. melas (different genus but same order) as a reference produced the minimum number of mate pairs $(7,048,651)$. Similar results were found for in silico mate-pair generation of $T$. bimaculatus using different references. Using T. rubripes and T. flavidus as references produced more mate pairs (T. rubripes : 268,610,220, T. flavidus : 386,830,523, respectively; same genus) than using $T$. nigroviridis as a reference $(10,334,324$, same family), while using $M$. mola as a reference genome produced the minimum number of mate pairs (M. mola : 1,059,534, same order).

The quantities of conserved mate pairs generated using two references (mag_mac**: 133,670,922) were greater than those obtained using three references (mag_mac_mel**: 4,474,485) (Table S7). Similar results were found for the number of conserved mate pairs generated for T. bimaculatus. Using four references (two from the same genus, one same family, and one same order) produced fewer number of mate pairs than using three references or two (rub-fla-nig-mol**: 360,635, rub-fla-nig**: 7,038,839, rub-fla**: 121,858,574) (Table S8). The number of conserved in silico mate-pair libraries with different insert sizes for different target species are shown (Tables S7-S9). The number of mate pairs was found to decrease with the application of more reference genomes.

\subsection{Effects of using different in silico mate pairs on genome assembly of C. batrachus}

The assemblies of C. batrachus generated using only paired-end libraries were unsatisfactory, the NGA50 only approximating $5.5 \mathrm{~Kb}$ and the number of complete BUSCOs (Benchmarking Universal Single-Copy Orthologs) 1,614 (Table 1). Both the original in silico method (mate pairs generated using one reference from the same genus) and the optimized in silico method (conserved mate pairs generated using two references from the same genus) significantly improved the genome assembly of $C$. batrachus . Compared to the original in silico method (using a single reference from the same genus, 'mag': C. magur or 'mac': C. macrocephalus ), the optimized in silico method (using two reference from the same genus, 'mag' and 'mac') reduced misassemblies (mag*:23,519; mac*: 25,442 vs. mag-mac**: 14,535), and yielded a similar NGA50 (mag*: $74.5 \mathrm{~Kb}$; mac*: $39.1 \mathrm{~Kb}$ vs. mag-mac**: $67.3 \mathrm{~Kb}$ ) and a similar number of complete BUSCOs (mag**:2,871; mac*: 2,659 vs. mag-mac**: 2,788$)$.

Compared to the original in silico method, optimized in silico method of generating conserved mate pairs using three reference genomes (two from the same genus 'mag', 'mac' and one from the same order 'mel') 
drastically decreased misassemblies (mag*:23,519; mac*: 25,442 , mel*:18,552 vs. mag-mac-mel**:7,671), but did not increase the NGA50 (mag*: $74.5 \mathrm{~Kb}$; mac*: $39.1 \mathrm{~Kb}$, mel*: $8.2 \mathrm{~Kb}$ vs. mag-mac-mel**: $5.5 \mathrm{~Kb}$ ) or complete BUSCOs (mag*:2,871; mac*: 2,659, mel*:1,756 vs. mag-mac-mel**: 1,618).

We compared the mate pairs generated using one reference genome (C. batrachus) with the conserved mate pairs generated using two reference genomes (C. batrachus and C. macrocephalus). We found that the extra mate pairs in the target genome generated using one reference were mostly inverted ( $45.76 \%$ to $47.21 \%$ ), while the remaining mate pairs in the target genome either displayed length deviations or were mapped to different scaffolds of the target genome (Table S11).

\subsection{Effects of using different in silico mate pairs on genome assemblies of $T$. bimaculatus}

Assembling the genome of $T$. bimaculatus, using only the paired-end reads yielded a NGA50 and a complete BUSCO number of $4.7 \mathrm{~kb}$ and 1,626, respectively, (Table 2). The original in silicomethod, as well as the optimized in silico method, improved the genome assembly of T. bimaculatus, significantly. Compared to the original in silico method (using one reference from the same genus, 'rub': T. rubripes or 'fla': T. flavidus ), the optimized in silico method (using two reference from the same genus, 'rub' and 'fla') increased the NGA50 (rub*: $140.2 \mathrm{~Kb}$; fla*: $131.4 \mathrm{~Kb}$ vs. rub-fla**: $183.8 \mathrm{~Kb}$ ) and reduced misassemblies markedly $\left(\mathrm{rub}^{*}: 5,143\right.$; fla*: 5,148 vs. rub-fla**: 4,188) with comparable number of complete BUSCOs (rub*:2,358; fla*: 2,366 vs. rub-fla**: 2,367$)$.

Compared to the original in silico method, the optimized in silico method which generated conserved mate pairs using more than two reference genomes (3 references: two from the same genus, 'rub', 'fla' and one from the same order, 'nig'; 4 references: using two reference from the same genus, 'rub', 'fla', one reference from the same family, 'nig', and one reference from the same order, 'mol') drastically reduced misassemblies (rub*: 5,143; fla*: 5,148, nig*: 5,843, mol*: 4,132 vs. rub-fla-nig**: 2,159, rub-fla-nig-mol*: 1,796), but failed to increase either the NGA50 (rub*: $140.2 \mathrm{~Kb}$; fla*: $131.4 \mathrm{~Kb}$, nig*: $7.2 \mathrm{~Kb}, \mathrm{~mol}^{*}$ : $4.7 \mathrm{~Kb}$ vs. rub-fla-nig**: $7.5 \mathrm{~Kb}$, rub-fla-nig-mol*: $4.6 \mathrm{~Kb}$ ) or the number of complete BUSCOs (rub*:2,358; fla*:2,366, nig*:1,772,

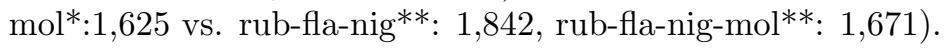

We compared the mate pairs generated using one reference genome (T. rubripes ) with the conserved mate pairs generated using two reference genomes (T. rubripes and $T$. flavidus ). We found that the extra mate pairs generated using one reference were mostly inverted on the target genome ( $60.03 \%$ to $66.62 \%)$, while the remaining mate pairs either had length deviation on the target genome or were mapped to different scaffolds of the target genome (Table S12).

\subsection{Genome assemblies of mountain nyala (degenerated DNA)}

Mate-pair generation of T. buxtoni, using B. grunniens as a reference, yielded the maximum number of mate pairs (B. grunniens : 416,044,705) while using $M$. moschiferus produced the least number of mate pairs $(M$. moschiferus : 220,576,118). The number of mate pairs generated using B. grunniens (same subfamily) as the reference genome was greater than that using T. scriptus and T. strepsiceros (same genus) as reference genomes (T. scriptus : 305,670,717, T. strepsiceros : 392,062,745), and this may be attributed to the high quality of B. grunniensassembly (Table S7-S9).

The mountain nyala ( T. buxtoni ) genome, which was generated with only paired-end reads from the degenerate samples, was not well assembled (Chen et al., 2019). The quality of the draft genome generated without using in silico mate-pair libraries was unsatisfactory (N50: $3.5 \mathrm{~Kb}$, complete BUSCOs: 645) (Table 3 ). Therefore, we used the original as well as the optimized in silico method to perform genome assembly of the mountain nyala. The results showed that when the original mate pairs were generated using different references ('scr': Tragelaphus scriptus, 'str': Tragelaphus strepsiceros, 'gru': Bos grunniens, 'mos': Moschus moschiferus), the draft genomes were improved, showing higher contiguity (N50-scr*: $592 \mathrm{~Kb}$, str*: $^{*} 31 \mathrm{~Kb}$, gru*:2.6 M, mos*:1.5 M) and increased completeness (Complete BUSCOs: scr*:1,956, str*:1,979, gru*:2,018, mos*:1,697). Compared to assemblies using the in silico method, genomes assembled using conserved mate pairs did not increase N50 (scr-str**: $203 \mathrm{~Kb}$, gru-scr**: $474 \mathrm{~Kb}$ ) or the number of complete BUSCOs (scr- 
str**: 1,727 , gru-scr**: 1,759$)$. Due to the low quality of the mountain nayala genome, no good reference genome could be used to calculate the misassembly rate.

\subsection{Testing optimized in silico method using simulated ancient DNA reads}

The quality of the genome assembly of $T$. flavidus generated using only short paired-end libraries was unsatisfactory (N50: $0.8 \mathrm{~Kb}$, complete BUSCOs: 148); (Table 4). When conserved in silicomate-pair libraries were generated using two genus references, compared to the original in silico mate-pair libraries using one reference, the NGA50 increased (NGA50: aDNA-rub-bim**: $438.4 \mathrm{~Kb}$ vs. aDNA-rub*:354.3 Kb), whereas misassemblies decreased significantly (misassemblies: aDNA-rub-bim**: 985 vs. aDNA-rub*: 1,661) and comparable numbers of complete genomes (complete BUSCOs: aDNA-rub-bim**: 2,156 vs. aDNA-rub*: BUSCOs: 2,205).

Genome assembly using the RaGOO pipeline showed higher contiguity (NGA50: aDNA-rub ${ }^{@}: 727.7$ Kb vs. aDNA-rub-bim**: $438.4 \mathrm{~Kb}$, @: assemblies using RaGOO method) and higher gene completeness (complete BUSCOs: aDNA-rub ${ }^{@}$ : 2,203 vs. aDNA-rub-bim**: 2,156), but with many more errors (misassemblies: aDNA-rub@: 1,829 vs. aDNA-rub-bim**:985), compared to using conserved in silicomate-pair libraries generated using two genera references. Synteny between assemblies and "real" genome (the best assembly of $T$. flavidus ) using the optimized in silico method was better than that using the RaGOO method (Figs. S2-S3).

\section{4 | DISCUSSION}

High-quality genome sequences are critical for biological research studies that focus on chromosomal structure and gene rearrangement, among others. Despite recent advances in sequencing technologies, many genome assemblies have not yet achieved the desirable level of quality. Forming the genome assemblies of some species with large or complex genomes poses challenges. Moreover, current technologies, such as long read sequencing and mate-pair sequencing, cannot be used to generate high-quality genome assemblies for some rare or extinct species, due to available DNA of these species being either degenerate or ancient. Therefore, in silico mate pair assembly may still be usable, especially for those species with only some degenerate DNA or ancient samples.

The phylogenetic distance to target species, quality, and completeness of the reference genome, as well as its overall synteny and transposable element content, affects the final quality of target genome assemblies. Thus, not all references are appropriate for genome assembly of a target species. Therefore, we tested multiple references with different phylogenetic distances to the genome assembly of the target species. This was demonstrated while constructing the genome assemblies of C. batrachus, T. bimaculatus, and T. buxtoni using in silico mate pair libraries that were generated using different references separately. In summary, a reference from the same genus as that of the target species is the best for making in silico mate pairs, compared with divergent references. In addition to phylogenetic distance, the quality of the reference genome also affected the target genome assembly. For example, the number of in silico mate pairs generated from the B. grunniens genome (different genera but same subfamily) to assemble the genome of T. buxtoni, was higher than those generated from T. scriptus or T. strepsiceros (same genus). The genome of B. grunniens had an N50 of $114 \mathrm{Mb}$, which was much larger than that of T. scriptus (890 Kb) or T. strepsiceros (511 Kb). Nevertheless, the number of complete BUSCO genes in the target genome assembled using B. grunniens as the reference was only slightly higher than that using the congener as the reference. Thus, the quality and completeness of references influence the final assemblies, but to a lesser extent than the influence of the phylogenetic distance of the reference species to the target.

Misassemblies, a common issue encountered in genome assembly, are mainly caused by sequencing or assembler errors. In de novo assembly based on long sequence reads, polishing with short reads is often used to improve the base-pair accuracy of assemblies (Rice \& Green, 2019). Misassemblies in reference-guide genome assemblers or scaffolders are inevitable due to unknown synteny and transposable element content discrepancies between the references and target species. This issue is particularly severe for assemblers that are designed based on one reference, which limits the wider use of reference-guide assembly algorithms or tools. 
Thus, the feasibility of reducing misassemblies in final genome assemblies is an important issue that needs to be explored by genomic studies. Therefore, we optimized the in silicomate-pair method by searching for conserved in silico mate pairs that reduce final misassemblies, under the assumption that conserved mate pairs would display more consistent synteny in the target species. We found that using three or more references (family or order conserved) reduced the number of misassemblies dramatically, but only by scarifying high contiguity and the number of complete genes. However, using two references from the same genus of the target species balanced contiguity, accuracy, and gene completeness of the final assemblies. By contrast, the original in silico mate-pair method using one reference resulted in more complete genes as well as in more misassemblies. Closer examination of these extra genes indicated that many did not exist in the "true" genome or were erroneous.

An increasing amount of sequence data of aDNA samples has been observed since the initial application of high-throughput sequencing to ancient human remains, (Rasmussen et al., 2010) over 2000 ancient samples being recorded (Brunson \& Reich, 2019). In addition to the limitations of aDNA sequences, such as read length and contamination, data processing and analysis algorithms lag behind current speeds and costs. This impedes paleogenomics, with particular reference to the recovery of the full nuclear genome. The genome assembly of ancient DNA data relies on the alignment of sequencing reads to a linear reference genome, leading to the selection of endogenous DNA sequences. Thus, we simulated aDNA sequences and used these for genome assembly via different methods. The results suggested that the optimized in silico mate-pair method performed better than the use of aDNA reads alone or the originalin silico mate-pair method. It also outperformed the assembler, RaGOO, in the level of accuracy, which may be attributed to the design of RaGOO, which is based only on one reference.

Use of in silico mate pairs for scaffolding is a simple method that enables long-range distance information from a reference genome to be incorporated into a de novo genome assembly, via the generation of in silico mate-pair libraries. It is essentially a novel reference-guide approach, since other chromosome scaffolders, such as Chromosomer (Tamazian et al., 2016), MeDuSa (Bosi et al., 2015), AlignGraph (Bao et al., 2014), and RaGOO (Alonge et al., 2019) exploit distance information from a genome of a closely related organism to order and extend scaffolds or contigs after the de novo assembly process. By contrast, in silico matepair libraries obtain distance information prior to the assembly process and can be adapted to any genome assembler that accepts mate-pair sequences as input. The contiguity of a genome assembly may be improved via the application of in silico methods or other reference-guided approaches. However, some referenceguided scaffolders rely heavily on paired-end or long-length read information, making these unsuitable for single-end reads. In addition, a large proportion of these reference-guided scaffolders are designed based only on one reference, resulting in many misassemblies in the draft genomes. Finally, all reference-guided genome assemblers or scaffolders have limitations, where only the conserved regions between target species and references are clear, while the sequence information between the conserved regions remains unknown.

\section{5 | CONCLUSION}

It is crucial that the in silico mate-pair method be used to assemble genomes from samples with only short fragment DNA, especially in the case of ancient DNA samples. Multiple reference genomes were used to select conserved mate-pair reads prior to assembling the genome. The contiguity and accuracy of genome assemblies were significantly improved. We suggest the following: (i) the closer the reference, the better the in silico mate-pair method; (ii) the optimized in silico mate-pair method should be used if two closely related references are available; and (iii) of the two reference genomes, the one with higher quality must be used as the first reference. This study provides guidelines for genome assembly using references and may benefit future genomic studies.

\section{DATA AVAILABILITY}

Custom scripts used for generating the results are available at GitHub (https://github.com/TaoZhou2021/optimized-insilico). Assembly of aDNA using the optimized in silico method is shown in the supplementary file. 


\section{ACKNOWLEDGEMENTS}

This work was supported by the "Science and Technology Commission of Shanghai Municipality (19050501900)" to CL.

\section{AUTHOR CONTRIBUTIONS}

CL and TZ conceived the research. TZ and LL assembled and simulated the data and performed the analysis. TZ, LL, and CL drafted the manuscript. All authors have edited and approved the final version of the manuscript.

\section{CONFLICT OF INTEREST}

The authors declare no competing interests.

\section{ORCID}

Tao Zhouhttps://orcid.org/0000-0002-5296-4237

Chenhong Lihttps://orcid.org/0000-0003-3075-1756

\section{REFERENCES}

Alonge, M., Soyk, S., Ramakrishnan, S., Wang, X., Goodwin, S., Sedlazeck, F. J., . . . Schatz, M. C. (2019). RaGOO: fast and accurate reference-guided scaffolding of draft genomes. Genome Biol, 20 (1), 224. doi:10.1186/s13059-019-1829-6

Bao, E., Jiang, T., \& Girke, T. (2014). AlignGraph: algorithm for secondary de novo genome assembly guided by closely related references.Bioinformatics, 30 (12), 319-328. doi:10.1093/bioinformatics/btu291

Berlin, K., Koren, S., Chin, C. S., Drake, J. P., Landolin, J. M., \& Phillippy, A. M. (2015). Assembling large genomes with single-molecule sequencing and locality-sensitive hashing. Nature Biotechnology, 33 (6), 623-630. doi:10.1038/nbt.3238

Boetzer, M., \& Pirovano, W. (2014). SSPACE-LongRead: scaffolding bacterial draft genomes using long read sequence information. BMC Bioinformatics, 15 (1), 211. doi:10.1186/1471-2105-15-211

Bongartz, P. (2019). Resolving repeat families with long reads.BMC Bioinformatics, 20 (1), 232. doi:10.1186/s12859-019-2807-4

Bosi, E., Donati, B., Galardini, M., Brunetti, S., Sagot, M. F., Lio, P., . . . Fondi, M. (2015). MeDuSa: a multi-draft based scaffolder.Bioinformatics, 31 (15), 2443-2451. doi:10.1093/bioinformatics/btv171

Brunson, K., \& Reich, D. (2019). The Promise of Paleogenomics Beyond Our Own Species. Trends in Genetics, 35 (5), 319-329. doi:10.1016/j.tig.2019.02.006

Chen, L., Qiu, Q., Jiang, Y., Wang, K., Lin, Z., Li, Z., . . . Wang, W. (2019). Large-scale ruminant genome sequencing provides insights into their evolution and distinct traits. Science, 364 (6446), eaav6202. doi:doi:10.1126/science.aav6202

de Man, T. J., Stajich, J. E., Kubicek, C. P., Teiling, C., Chenthamara, K., Atanasova, L., . . . Gerardo, N. M. (2016). Small genome of the fungus Escovopsis weberi, a specialized disease agent of ant agriculture. Proc Natl Acad Sci U S A, 113 (13), 3567-3572. doi:10.1073/pnas.1518501113

Gordon, D., Huddleston, J., Chaisson, M. J. P., Hill, C. M., Kronenberg, Z. N., Munson, K. M., . . . Eichler, E. E. (2016). Long-read sequence assembly of the gorilla genome. Science, 352 (6281), aae0344. doi:doi:10.1126/science.aae0344

Grau, J. H., Hackl, T., Koepfli, K. P., \& Hofreiter, M. (2018). Improving draft genome contiguity with reference-derived in silico mate-pair libraries. Gigascience, 7 (5). doi:10.1093/gigascience/giy029 
Gurevich, A., Saveliev, V., Vyahhi, N., \& Tesler, G. (2013). QUAST: quality assessment tool for genome assemblies. Bioinformatics, 29 (8), 1072-1075. doi:10.1093/bioinformatics/btt086

Iorizzo, M., Ellison, S., Senalik, D., Zeng, P., Satapoomin, P., Huang, J., . . . Simon, P. (2016). A high-quality carrot genome assembly provides new insights into carotenoid accumulation and asterid genome evolution. Nature Genetics, 48 (6), 657-666. doi:10.1038/ng.3565

Jain, M., Olsen, H. E., Paten, B., \& Akeson, M. (2016). The Oxford Nanopore MinION: delivery of nanopore sequencing to the genomics community. Genome Biol, 17 (1), 239. doi:10.1186/s13059-016-1103-0

Jarvis, D. E., Ho, Y. S., Lightfoot, D. J., Schmockel, S. M., Li, B., Borm, T. J. A., . . . Tester, M. (2017). The genome of Chenopodium quinoa. Nature, 542 (7641), 307-312. doi:10.1038/nature21370

Kolmogorov, M., Raney, B., Paten, B., \& Pham, S. (2014). Ragout-a reference-assisted assembly tool for bacterial genomes.Bioinformatics, 30 (12), i302-309. doi:10.1093/bioinformatics/btu280

Koren, S., Harhay, G. P., Smith, T. P., Bono, J. L., Harhay, D. M., McVey, S. D., . . . Phillippy, A. M. (2013). Reducing assembly complexity of microbial genomes with single-molecule sequencing. Genome Biol, 14 (9), R101. doi:10.1186/gb-2013-14-9-r101

Li, H. (2011). A statistical framework for SNP calling, mutation discovery, association mapping and population genetical parameter estimation from sequencing data. Bioinformatics, 27 (21), 2987-2993. doi:10.1093/bioinformatics/btr509

Li, H. (2013). Aligning sequence reads, clone sequences and assembly contigs with BWA-MEM. arXiv e-prints, arXiv:1303.3997.

Li, H. (2015). BFC: correcting Illumina sequencing errors.Bioinformatics, 31 (17), 2885-2887. doi:10.1093/bioinformatics/btv290

Lien, S., Koop, B. F., Sandve, S. R., Miller, J. R., Kent, M. P., Nome, T., . . . Davidson, W. S. (2016). The Atlantic salmon genome provides insights into rediploidization. Nature, 533 (7602), 200-205. doi:10.1038/nature17164

Loman, N. J., Quick, J., \& Simpson, J. T. (2015). A complete bacterial genome assembled de novo using only nanopore sequencing data.Nature Methods, 12 (8), 733-735. doi:10.1038/nmeth.3444

Luo, R., Liu, B., Xie, Y., Li, Z., Huang, W., Yuan, J., . . . Wang, J. (2012). SOAPdenovo2: an empirically improved memory-efficient short-read de novo assembler. Gigascience, 1 (1), 18. doi:10.1186/2047-217X-118

Marcais, G., Delcher, A. L., Phillippy, A. M., Coston, R., Salzberg, S. L., \& Zimin, A. (2018). MUMmer4: A fast and versatile genome alignment system. PLoS Comput Biol, 14 (1), e1005944. doi:10.1371/journal.pcbi.1005944

Marcais, G., \& Kingsford, C. (2011). A fast, lock-free approach for efficient parallel counting of occurrences of k-mers.Bioinformatics, 27 (6), 764-770. doi:10.1093/bioinformatics/btr011

Maretty, L., Jensen, J. M., Petersen, B., Sibbesen, J. A., Liu, S., Villesen, P., . . . Schierup, M. H. (2017). Sequencing and de novo assembly of 150 genomes from Denmark as a population reference.Nature, 548 (7665), 87-91. doi:10.1038/nature23264

Pop, M., Phillippy, A., Delcher, A. L., \& Salzberg, S. L. (2004). Comparative genome assembly. Briefings in Bioinformatics, 5 (3), 237-248. doi:10.1093/bib/5.3.237

Rasmussen, M., Li, Y., Lindgreen, S., Pedersen, J. S., Albrechtsen, A., Moltke, I., . . . Willerslev, E. (2010). Ancient human genome sequence of an extinct Palaeo-Eskimo. Nature, 463 (7282), 757-762. doi:10.1038/nature08835 
Rhoads, A., \& Au, K. F. (2015). PacBio Sequencing and Its Applications. Genomics Proteomics 8 Bioinformatics, 13 (5), 278-289. doi:10.1016/j.gpb.2015.08.002

Rice, E. S., \& Green, R. E. (2019). New Approaches for Genome Assembly and Scaffolding. Annual Review of Animal Biosciences, 7 (1), 17-40. doi:10.1146/annurev-animal-020518-115344

Sawyer, S., Krause, J., Guschanski, K., Savolainen, V., \& Paabo, S. (2012). Temporal Patterns of Nucleotide Misincorporations and DNA Fragmentation in Ancient DNA. Plos One, 7 (3). doi:10.1371/journal.pone.0034131

Schmieder, R., \& Edwards, R. (2011). Quality control and preprocessing of metagenomic datasets. Bioinformatics, 27 (6), 863-864. doi:10.1093/bioinformatics/btr026

Schneeberger, K., Ossowski, S., Ott, F., Klein, J. D., Wang, X., Lanz, C., . . . Weigel, D. (2011). Referenceguided assembly of four diverse Arabidopsis thaliana genomes. Proceedings of the National Academy of Sciences of the United States of America, 108 (25), 10249-10254. doi:10.1073/pnas.1107739108

Simao, F. A., Waterhouse, R. M., Ioannidis, P., Kriventseva, E. V., \& Zdobnov, E. M. (2015). BUSCO: assessing genome assembly and annotation completeness with single-copy orthologs. Bioinformatics, 31 (19), 3210-3212. doi:10.1093/bioinformatics/btv351

Smadbeck, J. B., Johnson, S. H., Smoley, S. A., Gaitatzes, A., Drucker, T. M., Zenka, R. M., . . . Vasmatzis, G. (2018). Copy number variant analysis using genome-wide mate-pair sequencing. Genes Chromosomes $\mathscr{E}$ Cancer, 57 (9), 459-470. doi:10.1002/gcc.5

Stoneking, M., \& Krause, J. (2011). Learning about human population history from ancient and modern genomes. Nature Reviews Genetics, 12 (9), 603-614. doi:10.1038/nrg3029

Tamazian, G., Dobrynin, P., Krasheninnikova, K., Komissarov, A., Koepfli, K. P., \& O'Brien, S. J. (2016). Chromosomer: a reference-based genome arrangement tool for producing draft chromosome sequences. Gigascience, 5 (1), 1-11. doi:10.1186/s13742-016-0141-6

Tan, Y. Q., Tan, Y. Q., \& Cheng, D. H. (2020). Whole-genome mate-pair sequencing of apparently balanced chromosome rearrangements reveals complex structural variations: two case studies. Molecular Cytogenetics, 13 (1), 15. doi:10.1186/s13039-020-00487-1

van Heesch, S., Kloosterman, W. P., Lansu, N., Ruzius, F.-P., Levandowsky, E., Lee, C. C., . . . Cuppen, E. (2013). Improving mammalian genome scaffolding using large insert mate-pair next-generation sequencing. BMC Genomics, 14 (1), 257. doi:10.1186/1471-2164-14-257

Wetzel, J., Kingsford, C., \& Pop, M. (2011). Assessing the benefits of using mate-pairs to resolve repeats in de novo short-read prokaryotic assemblies. BMC Bioinformatics, 12 (1), 95. doi:10.1186/1471-2105-12-95

TABLE 1 Statistics of the Clarias batrachus assemblies

\begin{tabular}{lllll}
\hline Assembly & Scaffold N50 (bp) & NGA50 (bp) & Misassemblies & Complete BUSCOs \\
\hline no_ in silico & 6,567 & 5,575 & 7,861 & 1,614 \\
mag* & 403,205 & 74,513 & 23,519 & 2,871 \\
mac* $_{\text {mel* }}^{130,451}$ & 283,737 & 39,183 & 25,442 & 2,659 \\
mag-mac** & 222,724 & 8,247 & 18,552 & 1,756 \\
mag-mac-mel** & 6,894 & 67,354 & 14,535 & 2,788 \\
\hline
\end{tabular}

Contiguity, accuracy, and BUSCO results of the Clarias batrachusassemblies using the original in silico method $(*)$ and optimizedin silico method $(* *)$. 'mag' short for 'Clarias magur ', 'mac' short for 'Clarias macrocephalus ', 'mel' short for 'Ameiurus melas'. no_in silico: without in silico method; *: original in 
silico method using one reference; **: optimized in silico method using multiple references.

TABLE 2 Statistics of the Takifugu bimaculatus assemblies

\begin{tabular}{lllll}
\hline Assembly & Scaffold N50 (bp) & NGA50 (bp) & Misassemblies & Complete BUSCOs \\
\hline no_ in silico & 7,103 & 4,695 & 1,601 & 1,626 \\
rub* & 940,637 & 140,231 & 5,143 & 2,358 \\
fla* & 858,358 & 131,404 & 5,148 & 2,366 \\
nig* $^{*}$ & 398,444 & 7,277 & 5,843 & 1,772 \\
mol $^{*}$ & 104,289 & 4,760 & 4,132 & 1,625 \\
rub-fla** & $1,275,322$ & 183,811 & 4,188 & 2,367 \\
rub-fla-nig** & 24,550 & 7,520 & 2,159 & 1,842 \\
rub-fla-nig_mol*** & 7,938 & 5,222 & 1,796 & 1,671 \\
\hline
\end{tabular}

Contiguity, accuracy, and BUSCO results of the Takifugu bimaculatus assemblies using the original in silico method (*) and optimized in silico method (**). 'rub' short for 'Takifugu rubripes', 'fla' short for 'Takifugu flavidus ', 'nig' short for 'Tetradon nigroviridis ', 'mol' short for 'Mola mola'. no_ in silico: without in silico method; *: original in silicomethod using one reference; ${ }^{* *}$ : optimized in silico method using multiple references.

TABLE 3 Statistics of the Tragelaphus buxtoni assemblies

\begin{tabular}{lll}
\hline Assembly & Scaffold N50 (bp) & Complete BUSCOs \\
\hline no_in silico & 3,561 & 645 \\
scr* & 592,242 & 1,956 \\
str* & 431,994 & 1,979 \\
gru* & $2,645,570$ & 2,018 \\
mos* & $1,518,369$ & 1,697 \\
scr-str** & 203,073 & 1,727 \\
gru-scr** & 474,151 & 1,759 \\
\hline
\end{tabular}

Contiguity and BUSCO results of the Tragelaphus buxtoniassemblies using the original in silico method $\left(^{*}\right)$ and optimizedin silico method (**). 'scr' short for 'Tragelaphus scriptus ', str' short for 'Tragelaphus strepsiceros ', 'gru' short for 'Bos grunniens ', 'mos' short for 'Moschus moschiferus '. no_ in silico: without in silico method; *: original in silico method using one reference; **: optimizedin silico method using multiple references.

TABLE 4 Statistics of the ancient DNA (Takifugu flavidus ) assemblies

\begin{tabular}{lllll}
\hline Assembly & Scaffold N50 (bp) & NGA50 (bp) & Misassemblies & Complete BUSCOs \\
\hline aDNA-no_in silico & 849 & - & 1,601 & 148 \\
aDNA-rub* $^{*}$ & $2,041,189$ & 354,329 & 1,661 & 2,205 \\
aDNA-rub $^{@}$ & $17,807,347$ & 727,701 & 1,829 & 2,203 \\
aDNA-rub-bim** $^{*}$ & $3,088,585$ & 438,498 & 985 & 2,156 \\
\hline
\end{tabular}

Contiguity, accuracy, and BUSCO results of the aDNA (Takifugu flavidus ) assemblies using the original in silico method $\left(^{*}\right)$ and optimized in silico method $\left({ }^{* *}\right)$. 'rub' short for 'Takifugu rubripes ', 'bim' short for 'Takifugu bimaculatus'. no_ in silico: without in silico method; *: original in silicomethod using one 
reference; **: optimized in silico method using multiple references; @: Ragoo method using one reference.

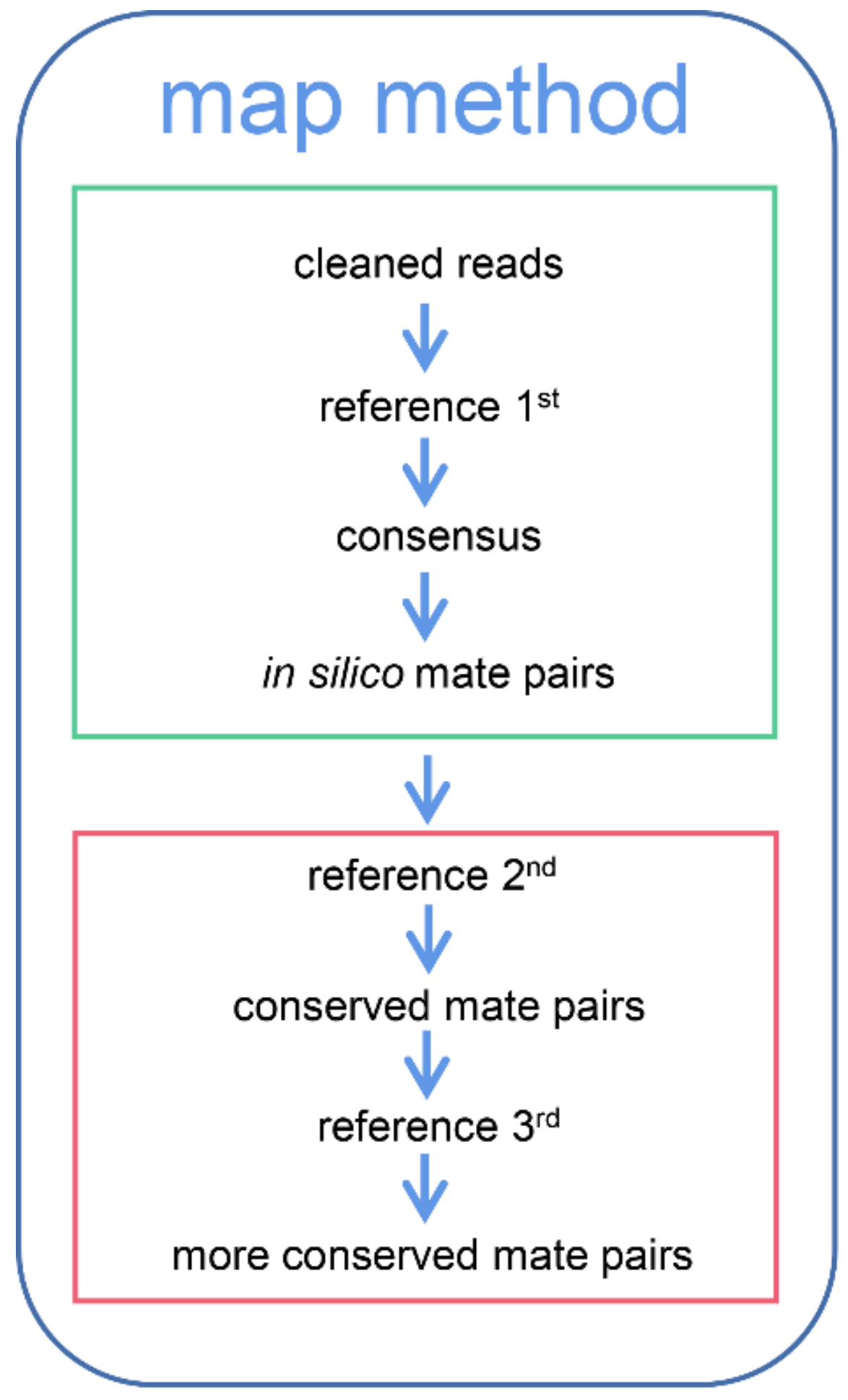

FIGURE 1. Scheme for original in silico mate-pair method and optimization (map method). The original in silico mate-pair method is shown in the green box, and the optimization is shown in the red box. 


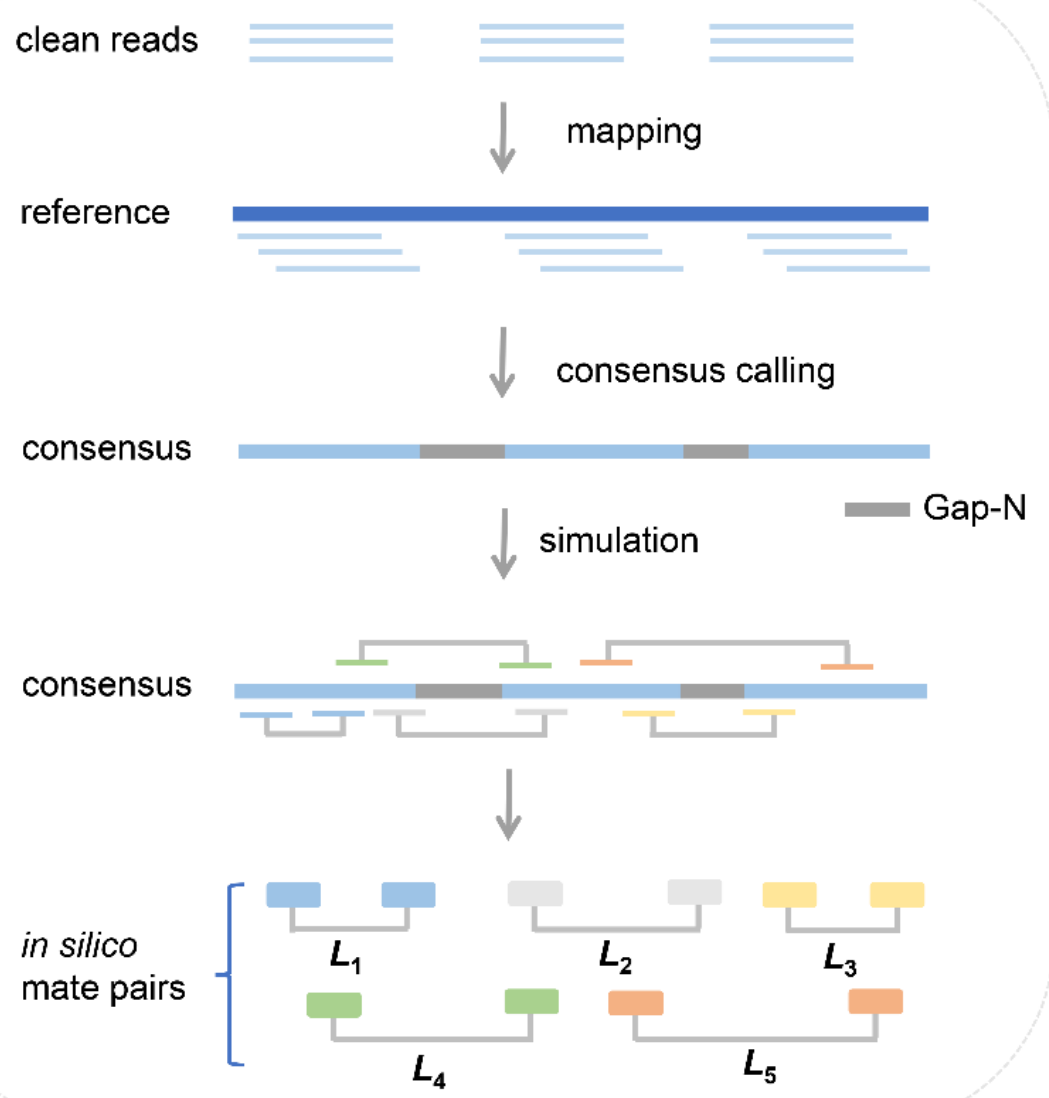

FIGURE 2. Scheme for generating in silico mate pairs. $L_{1}, L_{2}, L_{3}, L_{4}, L_{5} \ldots L_{\mathrm{N}}$ represent the different insert sizes of mate pairs.

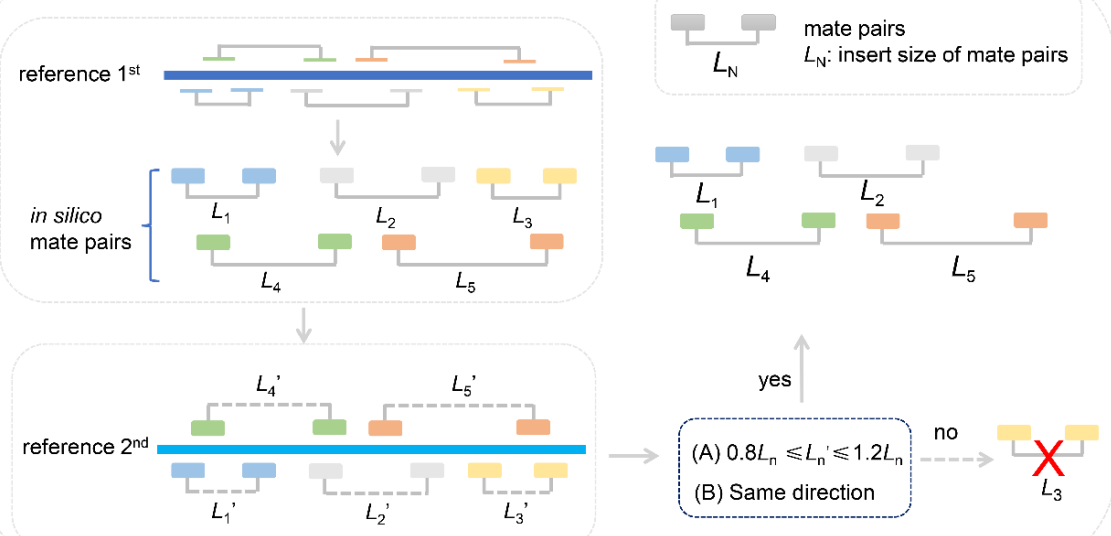

FIGURE 3. Scheme for optimization of in silico mate-pair method (map method). Mate pairs generated from the first $\left(1^{\text {st }}\right)$ reference were mapped to the second $\left(2^{\text {nd }}\right)$ reference. Only mapped mate pairs that 
satisfied the insert size of " $0.8 L \mathrm{n}$ [?] $L \mathrm{n}$ ' [?]1.2 $L \mathrm{n}$ " and that were also in the same direction were reserved for the following scaffolding process during genome assembly. 